\title{
Immunologic Desensitization
}

National Cancer Institute

\section{Source}

National Cancer Institute. Immunologic Desensitization. NCI Thesaurus. Code C15377.

Treatment or therapy to reduce or eliminate a patient's allergic response to an allergen $(\mathrm{NCl} / \mathrm{OSP})$ 